\title{
INDEX OF PASSAGES CITED
}

Aetius Amidenus

Iatricorum liber

16

15n39

Aeschylus

Agamemnon

$\begin{array}{ll}562 & 88 \\ 844 & 21 \mathrm{n} 1 \\ 848-850 & 21 \mathrm{n} 1 \\ 1001 \mathrm{ff} . & 76 \\ 1420 & 121 \mathrm{n} 2 \\ 1645 & 121 \mathrm{n} 2\end{array}$

Choephori

$183 \mathrm{f}$.

280

281

325

1017

1028

Eumenides

62

169

Persae (Persians)

196

415

416

715

Prometheus vinctus (Prometheus

Bound)

$377-380$

69

368

882

$94 \mathrm{n} 29$

73

Septem contra Thebas (Seven against Thebes)

682

Supplices

264

265

319

473

659
$121 n 2$

83

$121 n 2$

$121 n 2$

$121 \mathrm{n} 2$

56
Fragmenta

frag. 253

70

Alcmaeon of Croton

DK 24 B 4

$24 n 6$

Alexander of Aphrodisias

Problemata

$2.42 \quad 135 \mathrm{n} 25$

Commentary on Aristotle's Meteo-

rologica

CAG p. $67,12 \quad 163 n 9$

Anaximander

DK 12 A $27 \quad 163 n 9$

Anthologia Palatina

6.337

$113 n 5^{2}$

Archytas

DK 47 B $1 \quad$ 207n37, 209

Aretaeus (ed. Hude)

Causes and Signs of Acute Diseases

2.8.7 (p. 29,24 H.) 86n11, 88n14

Causes and Signs of Chronic Diseases

1(3).4.1 (p. 38,13 H.)

$88 \mathrm{n} 14$

1(3).5 (pp. 39-4o H.)

$243 n 44$

1(3).5 (p. 40,2 H.) 244n 47

$1(3) \cdot 5$ (p. 40,3-5 H.)

$245^{\mathrm{n}}{ }^{1}$

1(3).5 (p. 41,3f. H.)

$1(3) .14 .5$ (p. 57,3 H.)

$247 \mathrm{n} 57$

$88 \mathrm{n} 14$

2(4).10 (p. 79,15-30 H.)

$85 \mathrm{n} 9$

2(4).11 (p. 8o,23 H.)

84n4, 91n23 
Causes and Signs of Chronic Diseases

(cont.)

2(4).11 (p. 81,14 H.)

91022

2(4).11.7 (p. 81,16 ff. H.)

$84 \mathrm{n} 4$

Treatment of Acute Diseases

$$
\begin{gathered}
1(5) \cdot 1 \cdot 3 \text { (p. 92,4 H.) } 84 \mathrm{n} 4 \\
1(5) \cdot 9 \cdot 1 \text { (p. 113,9 H.) } \\
91123 \\
2(6) \cdot 3.12 \text { (p. 128,23 H.) } \\
184 \mathrm{n} 83
\end{gathered}
$$

Treatment of Chronic Diseases

$$
\begin{array}{r}
1(7) \cdot 7-8 \text { (p. 154,3-6 H.) } \\
129 n 17
\end{array}
$$

Aristophanes

Aves

$\begin{array}{ll}14 & 233 n 10 \\ \text { cclesiazusae } & \\ 216 \mathrm{f} . & 30 n 19 \\ 218-220 & 30 n 19 \\ 453 & 30 n 19 \\ 455^{-457} & 30 n 19 \\ 577 & 30 n 19 \\ 584 \mathrm{f} . & 30 n 19\end{array}$

Plutus

$\begin{array}{ll}12 & 233 \mathrm{n} 10 \\ 366 & 233 \mathrm{n} 10 \\ 636 & 68 \mathrm{n} 17 \\ 668 \mathrm{ff} . & 68 \mathrm{n} 20 \\ 903 & 233 \mathrm{n} 10 \\ \text { espae } & \\ 123 & 68 \mathrm{n} 17 \\ 1038 \mathrm{f} . & 63 \mathrm{ng}\end{array}$

Aristotle

Ars poetica

$\begin{array}{lc}\text { 1449b27 } & 55 \\ 145^{8 \mathrm{~b} 22} & 74 \\ \text { 1458b22-25 } & 90 \mathrm{n} 20 \\ \text { generatione } & \text { animalium } \\ 731 \mathrm{a} 24 & 309 \mathrm{ng} 2 \\ 743 \mathrm{~b} 22-25 & 309 \mathrm{n} 95 \\ 767 \mathrm{a} 2 \mathrm{f} . & 170 \\ 778 \mathrm{~b} 4 & 309 \mathrm{n} 94\end{array}$

De incessu animalium

$\begin{array}{ll}704 \mathrm{~b} 14 & 309 \mathrm{n} 94 \\ 704 \mathrm{~b} 15 & 309 n 93 \\ 711 \mathrm{a} 18 & 309 n 93\end{array}$

De partibus animalium

$\begin{array}{ll}\text { 1.1, 639b6 } & 309 n 94 \\ 1.1,639 \text { b19-21 } & 309 n 95 \\ 1.5,645 \text { a17 f. } & 309 n 96 \\ 1.5,645 \text { a24-25 } & 309 n 94 \\ 2.2,647 \text { b1off. } & 328 \\ 2.9,654 \text { b29 ff. } & 309 n 95\end{array}$

De sensu

$$
\text { 442b25 299n49 }
$$

Historia animalium

3.3, 512a12-513a7 315n3, 323n15

Meteorologica

38gb27-28 309n94

\section{Politica}

1286a12-14 12n3o

1268b26 ff. $\quad 36 \mathrm{n} 28$

1268b35 $\quad 36 \mathrm{n} 28$

1281b34f. $\quad 24$

1330a39-b18 $\quad 170$

Problemata

$3.1 \quad 179 n_{42}$

$3.6 \quad 179 n 42$

4.30, 880a3o-33 243n42

$7.8,887$ a3o $\quad 135 \mathrm{n} 25$

10.45895 b32-33 3ogng4

$30.1 \quad 237 \mathrm{n} 23,244 \mathrm{n} 45$

953a10-955a41 337n11

953a13 238n25

$953 \mathrm{a} 22 \quad 244 \mathrm{n} 48$

$953 \mathrm{~b} 6 \quad 238 \mathrm{n} 26$

953b24-25 241n34

954a12 240n29

954a21 241n32

954a21-26 240n30

954a3o-38 240n31

954a32 241n32

954a34-37 246n 54

954a35 246n 54

Rhetorica

1409a28ff. $\quad 47 \mathrm{n} 25$ 
Athenaeus

Deipnosophistae

1-2, 25 f. $-40 \mathrm{f}$.

$2.42 \mathrm{ab}$

$2.43 \mathrm{~b}$

$2.46, \mathrm{~b}-\mathrm{c}$

$2.46 \mathrm{c}$

$174 \mathrm{n} 6$

161

161

$171 \mathrm{n} 22$

171

Aulus Gellius

Noctes atticae

19.5

$170 n 19$

Beda Venerabilis

De temporum ratione

1.35

255n $82,25^{8 n} 61$

Caelius Aurelianus

Tardae passiones (Chronic Diseases)

$3 \cdot 3$

91022

Celsus

De medicina

$\begin{array}{ll}2.18 .12 & 171 \\ 2.11-13 & 174 \mathrm{n} 6 \\ 8 & 282 \mathrm{n} 65\end{array}$

Cicero

De finibus

4.4

28on57

Clemens Alexandrinus

Stromateis

$\begin{array}{ll}1.16 .75 & 19 n 49 \\ 6 & 69\end{array}$

Columella

De agricultura

$12.19 \mathrm{ff}$.

$174 n 6$

Critias

DK 88 B $25 \quad 102 n 15$

Democritus

DK 68 A 77

DK 68 B 191

DK 68 B 241

$212 n 5^{\circ}$

$32 \mathrm{n} 21$

$26 \mathrm{n} 12$

$91 \mathrm{n} 22$
Demosthenes

Contra Aristogitonem

$\begin{array}{lc}\begin{array}{l}95 \\ \text { Contra Cononem } \\ 3-4\end{array} & 91 \mathrm{n} 22 \\ & \\ & 178 \mathrm{n} 33\end{array}$

Diocles of Carystus

Fragmenta (ed. Wellmann/ ed. van der Eijk)

$$
\begin{array}{ll}
40 \mathrm{vdE} & 337 \mathrm{n} 13 \\
48 \mathrm{~W} .(114 \mathrm{vdE}) & 174 \mathrm{n} 7 \\
69 \mathrm{~W} .(120 \mathrm{vdE}) & 174 \mathrm{n} 7 \\
130 \mathrm{~W} .(237 \mathrm{vdE}) & 174 \mathrm{n} 7 \\
131 \mathrm{~W} .(238 \mathrm{vdE}) & 174 \mathrm{n} 7 \\
141 \mathrm{~W} .(182 \mathrm{vdE}) & 174 \mathrm{n} 7
\end{array}
$$

Diogenes Laertius

Vitae philosophorum

$\begin{array}{ll}3 \cdot 7 & 10 \text { 19 } \\ 7 \cdot 39 \text { and } 40 & 280 \text { 57 }\end{array}$

Diogenes Apolloniates DK 64 A $17 \quad 163 n 9$

Diodorus Sicilus

$\begin{array}{ll}1.82 .1-2 & 11 n 27 \\ 1.82 .3 & 12 \mathrm{n} 29 \\ 3.29 .6 & 94 \mathrm{n} 30 \\ 5.6 .3 & 94 \mathrm{n} 30 \\ 12.12 .3 & 94 \mathrm{n} 30 \\ 12.58 .3 & 129 n 16 \\ 14.51 .3 & 94 \mathrm{n} 30 \\ 14.54 \cdot 3 & 94 \mathrm{n} 30 \\ 17.26 .5 & 94 \mathrm{n} 30 \\ 20.96 .7 & 94 \mathrm{n} 30\end{array}$

Dioscorides

De materia medica

5

5.6

5.6 .2

174n6

5.6 .3

$180 n_{50}$

18 on 55

18on 52

5.6.10

$190 n 120$

5.6.11

1910133

Empedocles

DK 31 A $86 \quad 212$ 


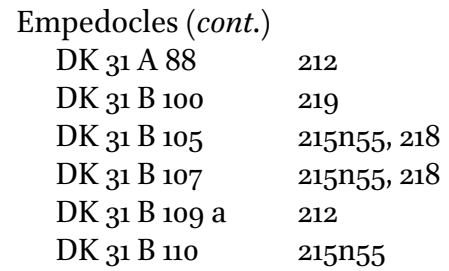

Epicurus

Epistola ad Herodotum

$\begin{array}{ll}37,2 & 298 \mathrm{n} 49 \\ 37,3 & 298 \mathrm{n} 49 \\ 78,2 & 298 \mathrm{n} 49\end{array}$

Erasistratus

Fragmenta (ed. Garofalo)

158

175n 7

164

$175^{\mathrm{n}} 7$

167

$175^{\mathrm{n}} 7$

283

$175^{\mathrm{n}} 7$

\section{Erotianus}

Hippocratic Glossary T4 (p. 84,7-11 Nachmanson)

85n8, 86n11, $87 \mathrm{n} 12$

M8 (p. 6o,1 Nachmanson)

$129 n 18$

Eupolis

Demes

frag. 94

$36 n 29$

Euripides

Alcestis

22

Antiope

frag. 201 v. 1-4

frag. 213

Autolycus

frag. 282 V. 4-9

Bacchae

1122-1123

Bellerophon

frag. 292

Hercules Furens

1233

65n10, 78

$121 n 2$
1324

Heracleidae

$55^{8}$

Hippolytus

35

317

141-148

159

$161 \mathrm{f}$.

179

186

$198 \mathrm{f}$.

946

1029

Iphigenia in Aulis

281-283

284

299

Iphigenia in Tauris

946

1047

1178

1226

Medea

1173-1175

1189

1187

$1200 \mathrm{ff}$.

$1268 \mathrm{f}$.

Orestes

$34 \mathrm{ff}$.

226

234

387

517

523

524

598

Philoctetes

frag. 792 Nauck 9on19, 91022

Phoenissae

816

$121 \mathrm{n} 2$

892-893

$21 \mathrm{n} 1$

Supplices

$201 \mathrm{ff}$.

252

12112

$121 n 2$

121n 2

$121 \mathrm{n} 2$

104n21

64

64

76

77

76

121n2

67

73

73

72

$121 n 2$

$121 n 2$

$121 n 2$

$121 n 2$

73

89, 9on19

95

89,95

$121 \mathrm{n} 2$

82, 82n2

$33 n 24$

82

$121 n 2$

96n33

96n33

121n2
$82,83 n 2,96 n 33$ 
Fragmenta (ed. Nauck)

$\begin{array}{ll}\text { frag. } 78 & 23 n 4 \\ \text { frag. } 292 & 110 \text { 40 } \\ \text { frag. } 792 & 70 \\ \text { frag. } 917 & 69,78 \\ \text { frag. } 981 & 78 \\ \text { frag. } 1072 & 78\end{array}$

Galen and Pseudo-Galen

Ad Pisonem de theriaca liber

14.277,4 K. 231n 4

16 (14.281,8-18 K.)

Ars medica

$279 n 54$

1.326,9-329,10 K. $338 \mathrm{n} 16$ 21 (1.358,7-9 K.) 296n36 1.378,9-10 K. $311 n 106$

De alimentorum facultatibus 3.40 (6.743,1 ff. K.)

$3.40(6.744,3-5$ K.) $183 n 73$ $184 n 81$

De anatomicis administrationibus

$5.4(2.502,9 \mathrm{~K}) \quad .302 \mathrm{n} 62$

$6.1(2.542,13$ K. $) \quad 295 n 33$

$6.1(2.543,17$ K.) $\quad 295 n 33$

6.3 (2.545,6f. K.) $\quad 295$ n33

De antidotis

1.3 (14.14-20 K.) 1900119

De atra bile

7 (5.131,12 K.) $302 n 62$

8.9 (p. 93,16-18 de Boer)

2310

De bonis et malis alimentorum sucis

$11(6.803,1$ K. $) \quad 184 n 80$

$11(6.803,11 \mathrm{ff} . \mathrm{K}) \quad .181 \mathrm{n} 62$

$11(6.808,4-7$ f. K. $)$

$181 n 61$

De causa affectionum

p. $18 \mathrm{f}$. Helmreich $125 \mathrm{n} 9$

De causis procatarcticis

(Antecedent Causes)

$100 \quad 134 n 22$

108 (CMG Suppl. II, p. 26)

133n22
De compositione medicamentorum per genera (The Composition of Drugs According to Kinds)

$5.1(13.776,18 \mathrm{f.} \mathrm{K}) \quad 18 \mathrm{n} 46$

$5.1(13.778,7$ f. K) $\quad 18 \mathrm{n} 46$

7.1 (13.950,10-12 K.)

296n39

De compositione medicamentorum secundum locos (The Composition of Drugs According to Places)

1.1 (12.379,8-380,9 K.) 293n29

$1.1(12.381,4-7$ K.) $265 \mathrm{n} 10,265 \mathrm{nu}$

$4.8(12.749,14 \mathrm{~K}) \quad .18 \mathrm{n} 44$

$6.8(12.965,11-15$ K.) $265 \mathrm{n} 12$

De constitutione artis medicae ad Patrophilum liber

$1(1.227,10 \mathrm{ff}$. K. $) \quad 310 \mathrm{nio2}$

$6(1.244,13-15$ K.) 28on55

De consuetudinibus

1 (p. 12,15-16,4 Mueller)

1 (p. 9,15-18 Mueller) $149 n 33$

De febrium differentiis $149 n 33$

$1.3(7.279,11-13 \mathrm{~K}$.

$135 \mathrm{n} 25$

De febrium differentiis (cont.)

$1.6 \quad 130,133 n 22$

$1.6(7.289,4-290,11$ K.)

$7.289,18 \mathrm{~K} . \quad 133 \mathrm{n} 22$

$7.290,10 \mathrm{~K} . \quad 133 \mathrm{n} 22$

7.291,2 f. K. $\quad 133 n 22$

7.291,3f. K. $\quad 133 \mathrm{n} 22$

7.291,17f. K. $\quad 133 \mathrm{n} 22$

De humoribus

$9\left(19.492,15^{-493,1}\right.$ K.)

De libris propriis

$347 n 38$

6 (p. 114,2-5 Müller)

6 (p. 114,2 ff. Müller)

$275^{\mathrm{n}} 40$

$275^{n} 41$

6 (p. 113,13-18 Müller) 


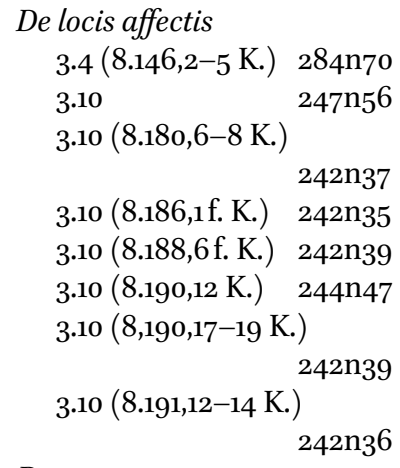

De marcore

1.2 (7.669,6 ff. K.) 295n34

De naturalibus facultatibus

$$
\begin{aligned}
& 1.10(2.23,11 \mathrm{~K} .) \quad 309 n 91 \\
& 1.12(2.27,1-3 \text { K. }) \quad 307 n 81 \\
& 1.13(2.38,4-39,6 \text { K.) } \\
& 301559 \\
& 1.13 \text { (2.38,10 ff. K.) 30on58 } \\
& 1.13(2.40,9 \text { K.) } 231 \mathrm{nn} 4 \\
& 2.3(2.80,6-93,13 \mathrm{~K} \text {. }) \\
& 2.4(2.88,14-89,4 \text { K.) } \\
& 308 \mathrm{n} 86 \\
& \text { 3oon } 55 \\
& 2.4(2.90,2-3 \text { K. }) \quad 308 \text { ngo } \\
& 2.4 \text { (2.91,8ff. K.) } 308 \mathrm{n} 85 \\
& 2.4(2.92,14-15 \mathrm{~K} \text {. }) \\
& 2.4(2.92,14-16 \text { K.) } \\
& 308 n 89 \\
& 3^{300 n_{5} 6} \\
& 2.9(2.131,11 \text { K. }) \quad 309 n 91 \\
& 2.9(2.132,3-8 \text { K.) } 337 n 14 \\
& 2.9(2.141,5 \text { f. K. }) \quad 337 \mathrm{n} 12
\end{aligned}
$$

De optima corporis nostri constitutione

$$
2 \text { (4.741,10 f. K.) 328n19 }
$$

De optimo medico cognoscendo

5.1 (p. 69,4-7 Iskandar) 284n67

9.18 (p. 113,8-11 Iskandar) 279n 52

De placitis Hippocratis et Platonis

$$
\begin{array}{r}
6.1 .3(5.506,6 \text { f. K. }) \\
292 \mathrm{n} 24 \\
6.1 .8-9\left(5 \cdot 5^{07,12-18} \text { K. }\right) \\
291 \mathrm{n} 22
\end{array}
$$

$\begin{array}{ll}8.4 .21-23 & 340 n 21 \\ 8.6 .17 & 339 n 19\end{array}$

9.5.1 ff. (5.750-751 K.) $281 n 60$

9.8.26-27 (5.790,16-791,8 K.) 301559

$9.8(5 \cdot 791,2-3$ K. $) \quad 311 n 106$ De praenotione ad Epigenem (On Prognosis)

5 (14.625,8-11 K.) 284n69

8 (14.647,12 K.) 284n68

De purgantium medicamentorum facultate

1 (11.323,1ff. K.) 285n71

De tumoribus praeter naturam

(On Tumours contrary to Nature)

$$
\text { 7.727,7-9 K. } \quad 90 \mathrm{n} 22
$$

De sanitate tuenda

2.5 (6.334,6f. K.) $\quad 181 n_{59}$

6.2 (6.384,4f. K.) $327 \mathrm{n} 18$

De simplicium medicamentorum temperamentis ac facultatibus

6, prol. (11.792,12 f. K.)

$$
\begin{array}{ll}
7.15 .2(12.88 \text { K.) } & 17 \mathrm{n} 41 \\
8.4(12.179,6 \text { K. }) & 92 \mathrm{n} 22
\end{array}
$$

De symptomatum causis $2.7(7.202,18-203,3 \mathrm{~K}$.)

$$
242 n 40
$$

De temperamentis

$1.1 \mathrm{ff}$. (1.509 ff. K.) $338 \mathrm{n} 16$

2.5 (1.619,8ff. K.) $292 \mathrm{n} 26$

$2.5(1.619,10-14$ K.)

$293 n 27$

2.6 (1.630,12 ff. K.) $338 \mathrm{n} 17$

2.6 (1.641,7 f. K.) $338 \mathrm{n} 18$

De usu partium

$1.8(3.16,7-9$ K.) $\quad 299 n 52$

$1.8(3.16,10-12$ K.) $309 n 98$

1.8 (ed. Helmreich 1.11)

$\begin{array}{ll}1.8(3.17,14 \text { ff. K. }) & 33^{2 n} 20 \\ 1.18(3.64,14 \text { K. }) & 309 n 91 \\ 1.22(3.81,10 \text { f. K. }) & 304 n 70 \\ 2.8(3.122,2 \text { K. }) & 303 n 66 \\ 2.12(3.137,9 \text { K. }) & 309 n 91 \\ 3.6(3.193,18 \text { K. }) & 309 n 91\end{array}$




$$
\begin{aligned}
& 3.10(3.233,10-11 \mathrm{~K} .) \\
& 3.10\left(3.235,6-8 \text { K.) } 304 \mathrm{n}^{3041}\right. \\
& 3.10(3.237,11 \text { K. }) \quad 303 n 68 \\
& 4.13(3.301,3 \mathrm{~K} .) \quad 303 n 65 \\
& 5 \cdot 3(3 \cdot 350,16-351,2 \text { K.) } \\
& \text { 294n3o } \\
& 6.21(3.511,13 \text { K. }) \quad 302 \mathrm{n} 62 \\
& 7.14(3.572,2 \mathrm{~K} .) \quad 302 n 67 \\
& 7.22(3.607,13 \text { K. }) \quad 302 n 62 \\
& 11.14 \text { (3.907,8ff. K.) } \\
& \text { 293n28 } \\
& 12.14(4.56,13 \text { K. }) \quad 308 \mathrm{n} 84 \\
& 13.2 \text { (4.78,6f. K.) } \quad 308 \mathrm{n} 84 \\
& 13.8 \text { (4.112,6f. K.) } \quad 308 \mathrm{n} 84 \\
& 15.4(4.228,14 \text { K. }) \quad 308 \mathrm{n} 84 \\
& 15.5(4.240,17 \text { K. }) \quad 308 n 84 \\
& 16.14 \text { (4.343,12 f. K.) } \\
& 302 n 61 \\
& 17.1 \text { (4.36o,15-361,2 K.) } \\
& 310 n 101 \\
& \text { Definitiones medicae } \\
& 65 \text { (19.363,14-364,3 K.) } \\
& 104 \text { (19.374,2-9 K.) } \\
& 35^{6} n_{53} \\
& 35^{6 n} 53 \\
& \text { 19.416,9-13 K. } \quad 247 \text { n } 56 \\
& \text { 19.419,3-7 K } 91 \mathrm{n} 22 \\
& 19.443,3 \text { ff. K. } \quad 91 \mathrm{n} 22 \\
& 462 \text { (19.457,13-16 K.) }
\end{aligned}
$$

In Hippocratis Aphorismos commentarii

$$
17 \text { B.529 K. 288n6, 288n8, }
$$

\begin{tabular}{|c|c|}
\hline p. $3^{-11} \mathrm{M}$. & 316 \\
\hline 3,4-19 M. & 318 \\
\hline p. $3,20-7,14 \mathrm{M}$. & 324 \\
\hline p. $6,15 \mathrm{M}$. & $327 \mathrm{n} 18$ \\
\hline . $7,19-8,18 \mathrm{M}$. & $3^{20 n 11}$ \\
\hline . 8,10 M. & 321 \\
\hline p. $8,17 \mathrm{M}$. & $3^{21}$ \\
\hline p. $8,19-9,11 \mathrm{M}$. & 329 \\
\hline 1.1 & $338 n 15$ \\
\hline $1.1(15 \cdot 7,7$ f. K. $)$ & $287 \mathrm{n} 1$ \\
\hline 1.32 & 339 n20 \\
\hline 1.40 & $340 n 23$ \\
\hline $1.42(15.103,11 \mathrm{~K})$. & $2995^{2}$ \\
\hline p. $53,17-55,25 \mathrm{M}$. & 330 \\
\hline pp. $53-56 \mathrm{M}$. & 316 \\
\hline p. $57 \mathrm{M}$. & 316 \\
\hline p. $57,12-20 \mathrm{M}$. & 321 \\
\hline p. 62 f. M. & $134 \mathrm{n} 23$ \\
\hline p. $63,18-20 \mathrm{M}$. & $125^{n g}$ \\
\hline p. $69,30-70,17 \mathrm{M}$. & 322 \\
\hline p. $70,17-23 \mathrm{M}$. & 322 \\
\hline p. $73,18 \mathrm{M}$. & 32 \\
\hline p. 75 & \\
\hline 0.05 & \\
\hline
\end{tabular}$$
288 \mathrm{n} 10
$$$$
17 \text { B.529-532 K. } 288 \text { n3 }
$$$$
17 \text { B. } 532 \text { K. } 290 n 18
$$$$
17 \text { B. } 532,6-8 \text { K. } \quad 289 n 14
$$$$
17 \text { B.532,10 K. } \quad 289 n 15
$$$$
17 \text { B. } 532,15 \text { f. K. } \quad 289 n 16
$$$$
17 \text { B. } 537 \text { K. } \quad 271029
$$$$
17 \text { B.565 K. } 288 n_{5}, 288 n 7 \text {, }
$$$$
\text { 288ng, 288n12 }
$$$$
17 \text { B. } 565-566 \text { K. } \quad 288 \text { n } 4
$$$$
17 \text { B.659,15 K. 231n4 }
$$$$
17 \text { B.659,9-66o,3 K. }
$$

$243 n 41$

In Hippocratis de acutorum morborum victu

$$
\begin{aligned}
& \text { 15.570,1-4 K. 288n11 } \\
& 3.1(15.626,4 \text { f. K.) } 179 n 46 \\
& 3.2(15.633,9 \mathrm{ff} . \mathrm{K} .) \\
& 179 n 39 \\
& 3.2(15.633,14-17 \text { K.) } \\
& \text { 18on } 56 \\
& 3.7 \text { (15.646,10 f. K.) } \\
& \text { 180n53 }
\end{aligned}
$$

De elementis secundum Hippocratem

$1.2(1.415,4-10$ K. $) 299 n_{51}$

$1.5(1.449,2-4$ K. $) \quad 308 \mathrm{n} 88$

$1.9(1.486,10$ f. K.) 299n54

$1.9(1.487,8$ ff. K. $) \quad 298 \mathrm{n} 47$

$1.9(1.487,8-9$ K. $) \quad 299 n 53$

$1.9(1.487,12-14 \mathrm{~K}$.

$$
298 n 44
$$

In Hippocratis de natura hominis

(Commentary on Hippocrates' Nature of Man) (ed. Mewaldt)

Prooemium 
In Hippocratis epidemiarum librum primum commentarii III

$$
\begin{array}{ll}
2.5^{0} \text { (17A.148f. K.) } & \\
& 264 \mathrm{ng} \\
2.51 \text { (17A.15o f. K.) } & 266 \mathrm{n} 14 \\
3.7 \text { (17A.239 K.) } & 133 \mathrm{n} 22
\end{array}
$$

In Hippocratis epidemiarum librum secundum commentarii $V$

$$
\text { p. 16o Pfaff 86n11 }
$$

In Hippocratis Epidemiarum librum VI commentaria I-VI

p. 89 Wenkebach-Pfaff

$86 n 11$

17A.994-995 K. $\quad$ 272n35

17B.29,10 K. 241n34

17B.29,13-17 K. $\quad 243 n 42$

17B.135f. K. $\quad 271029$

${ }_{17}$ B.135,4ff. K. $\quad 270 n 26$

17B.136,12 f. K. $\quad 270 n 28$

${ }_{17}$ B.136,14 K. $\quad 271 n 30$

${ }_{17} \mathrm{~B} .142,8 \mathrm{~K} . \quad 272 \mathrm{n} 32$

${ }_{17}$ B.144-152 K. 268 n 20

${ }_{17}$ B.146,7.13 K. $\quad 269 n 21$

17B.147,4-6 K. $\quad 267 \mathrm{n} 17$

17B.147,16-18 K. $\quad 268$ n19

17B.148,13f. K. $\quad 269 n 22$

17B.149,1-7 K. $\quad 269 n 24$

$4.13 \quad 290018$

17B.222-233 K. $310 n 104$

17B.223,6.8 K. $291 \mathrm{n} 21$

17B.231,12 f. K. $\quad 311 n 107$

17B.233,6 f. K. $311 n 107$

17B.240,12 K. $\quad 302 \mathrm{n} 62$

${ }_{17}$ B.266-269 K. $\quad 272$ n33

$5.1 \quad 290017$

17B.287,7 K. $\quad 302 n 62$

In Hippocratis Prognosticon commentaria III

4 = pp. 205-209 Heeg

$111 n_{42}$

In Hippocratis prorrheticum I commentaria III

$1.25 \quad 84 \mathrm{n} 6$

Introductio sive medicus

ch. 9

19

9 (14.695,8-696,13 K.)

$35^{6 n} n_{53}$ $13\left(14.726,2-6\right.$ K.) $35^{6 n} 53$

$13(14.727,10-14$ K.) $35^{6 n} 53$

$13(14 \cdot 730,17-731,1 \mathrm{~K}$. $35^{6} \mathrm{n}_{53}$

14,740-741 K. $\quad 246$ n 55

14.674-676 K. $\quad 16 \mathrm{n} 4 \mathrm{o}$

14.674-797 K. $15 \mathrm{n} 38$

$14.706,12-15 \mathrm{~K} . \quad 15 \mathrm{n} 39$

Linguarum seu dictionum exoletarum Hippocratis explicatio

19.103,12 K. $\quad 85,85 n 9,86 n 11$

$19 \cdot 103,12-104,5$ K. 85 n8

19.134,2 K. 83

Methodus medendi

$1.2(10,13$ f. K.) $\quad 332 \mathrm{n} 20$

1.2 (10.15,8f. K.) $307 \mathrm{n} 82$

$1.2(10.16,8 \mathrm{~K}) \quad .307 \mathrm{n} 83$

$1.2(10.16,13-16 \mathrm{~K}$.

$310 n 100$

$1.2(10.17,2-4$ K. $) \quad 308 n 87$

11.15 (10.783,8 ff. K.)

$278 \mathrm{n}_{51}$

Quod animi mores corporis temperamenta sequuntur

$$
\begin{array}{ll}
1.3 & 340 \mathrm{n} 23 \\
1.3(4.776,17-777,4 & \text { K. }) \\
& 340 \mathrm{n} 22 \\
1.8(4.779,5-7 \text { K.) } & 340 \mathrm{on} 22
\end{array}
$$

Quod optimus medicus sit quoque philosophus (That the Best Doctor is also a Philosopher) (ed. Müller)

ch. 2 $276,277,283$

p. $2,21-3,6 \mathrm{M} . \quad 283 n 67$

p. $3,6-8 \mathrm{M} . \quad 276 \mathrm{n} 43$

p. 3,18-4,2 M. $\quad 276 \mathrm{n} 44$

p. 3,19 f. M. $\quad 281 n_{59}$

p. $4,3-7$ M. $277 \mathrm{n} 45$

p. 4,18-21 M. 277n 46

p. 5,6-12 M. 277n 47

p. 5,12-6,6 M. 279n 54

p. $6,4-10 \mathrm{M} . \quad 279 n 53$

p. 6,14-19 M. 300n57, 333

ch. $3 \quad 277$

ch. $4 \quad 282$

Thrasybulus

30 (5.861f. K.) $\quad 310 n 102$ 


$$
\begin{array}{ll}
30 \text { (5.862,5-7 K.) } & 311 n 106 \\
32(5.869 \text { K. }) & 17 n 42
\end{array}
$$

\section{Gorgias \\ DK 82 B $8 \quad 5^{1}$ \\ DK 82 B 11 a $\quad 23 n 4$ \\ DK 82 B11 (2) 49n34 \\ DK 82 B 11 (5) $\quad 50{ }^{3} 36$ \\ DK 82 B11 (8) $\quad 49 n 33$ \\ DK 82 B 11(14) 39n2 \\ DK 82 B $11(21) \quad 49 n 35$}

Heraclitus

$$
22 \text { B } 5 \text { DK } \quad \text { 108n33 }
$$

\section{Herodotus}

$\begin{array}{ll}1.105 & 106,106 \mathrm{n} 23 \\ 1.133 & 141 \\ 2.77 & 11 \mathrm{n} 24 \text { and } 26, \\ & 32 \mathrm{n} 21 \\ 2.84 & 10 \mathrm{n} 20 \\ 3.1 & 10 \mathrm{n} 21 \\ 3 \cdot 33 & 99,99 \mathrm{n} 3 \\ 3.82 & 35 \mathrm{n} 28 \\ 3.129 & 10 \mathrm{n} 22,13 \mathrm{n} 32 \\ 3.130 .3 & 14 \mathrm{n} 33 \\ 3.131 & 5^{2 \mathrm{n} 42} \\ 3.133 & 94 \\ 4.67 & 106 \\ 5.101 & 94 \\ 7.120 & 144 \mathrm{n} 22 \\ 7.171 & 58 \mathrm{n} 5 \\ 9.82 & 143 \mathrm{n} 18\end{array}$

Herophilus

T 256 (von Staden)

$175^{\mathrm{n}} 7$

Hesiod

$\begin{array}{ll}\begin{array}{l}\text { Opera et Dies } \\ 238 \mathrm{ff} .\end{array} & \\ 240 & 58 \\ 243 & 56 \\ 255 \mathrm{f} . & 5^{6} \\ 596 & 189 n 109\end{array}$

Hippocraticum Corpus

Aphorismi

$$
\begin{array}{ll}
1.1(4.458,2-4 \text { L. }) & 267 \mathrm{n} 18 \\
1.2(4.458 \text { L. }) & 69 \\
1.3(4.458,11 \text { f. L. }) & 76 \\
1.17(4.468,1 \text { f. L. }) & 148,148 \mathrm{n} 31 \\
2.21(4.476,5 \text { L. }) & 183 n 72 \\
2.29 & 149 n 32 \\
2.34 & 288 \mathrm{n} 3 \\
2.38(4.480,17 \text { f. L. }) \\
\multicolumn{2}{c}{270 \mathrm{n} 27}
\end{array}
$$$$
2.49(4.484,3-5 \text { L. })
$$$$
\text { 25, 26n12 }
$$$$
2.50 \quad 27,34,35
$$$$
3.2 \quad 288 \mathrm{n} 4
$$

3.20 (4.494,16f. L.) $236 n 21$

$3.22(4.496,7$ f. L.) $236 \mathrm{n} 21$

$5.22(4.540,3$ L. $) \quad 91 n 23$

5.26 (4.542,1-2 L.)

$161 n 6$

6.23 (4.568,11f. L.)

$235 \mathrm{n} 15$

6.56 (4.576,19f. L.)

$$
\begin{aligned}
& \text { 235n19 } \\
& 7.40 \text { (4.588,8f. L.) } 235 \mathrm{n} 19 \\
& 7.48 \text { (4.590,10 f. L.) }
\end{aligned}
$$

Coacae praenotiones

$\begin{array}{ll}84 \text { (5.602,5 L. }) & 84 \mathrm{n} 5 \\ 151(5.616,6 \text { L. }) & 84 \mathrm{n} 5 \\ 241(5.636,14 \text { L. }) & 84 \mathrm{n}_{5}, 87 \mathrm{n} 13 \\ 458 & 85 \mathrm{ng} \\ 459 & 85 \mathrm{ng} \\ 613\left(5 \cdot 726,13^{-17} \text { L. }\right) & 85 \mathrm{n} 10,87 \\ 613(5 \cdot 726,17 \text { L. }) & 84 \mathrm{n} 5,85 \mathrm{n} 10\end{array}$

De aere, aquis, locis

$\begin{array}{ll}1 & 144 \mathrm{n} 21 \\ 1(2.12,1 \text { L. }) & 45^{\mathrm{n} 16} \\ 1(2.12,6-9 \text { L. }) & 159 \mathrm{n}, 161 \mathrm{n} 7 \\ 1(2.12,18-21 \text { L. }) & 139 n 8 \\ 2(2.14,9 \text { f. L. }) & 3^{2 \mathrm{n} 21} \\ 2(2.14,10 \text { L. }) & 3^{2 \mathrm{n} 21} \\ 2(2.14,16-18 \text { L. }) & 15^{8} \\ 2(2.14,18 \text { L. }) & 3^{2 \mathrm{n} 21}\end{array}$




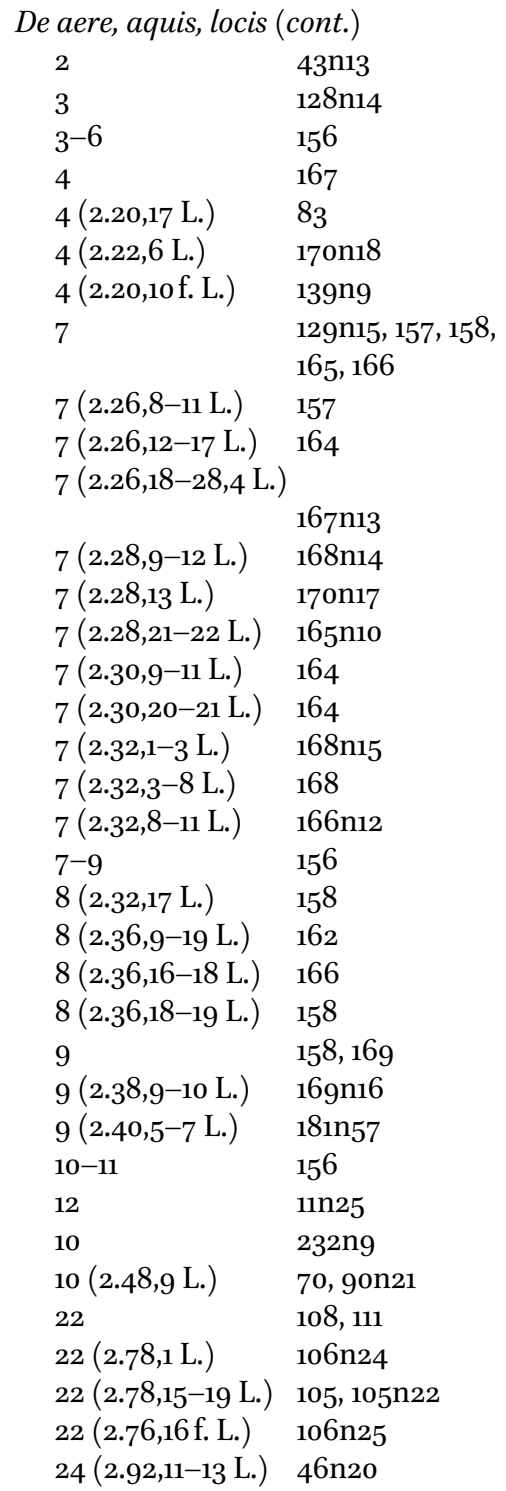

$$
\begin{aligned}
& 38 \text { (6.248,8 L.) } \quad 192 n 140 \\
& 40(6.250 \text { L. }) \quad 186 \mathrm{ng}^{2} \\
& 40 \text { (6.25o,9f. L.) } \quad 187 \mathrm{ng} \\
& 40 \text { (6.25o,10f. L.) } \quad 184 \mathrm{n}_{74} \\
& 42 \text { (6.252,1 L.) } \quad 192 \mathrm{n} 147 \\
& 43 \quad 144 \text { n21 } \\
& \text { 47-6o (6,254-269 L.) } \\
& 48 \text { (6.258,16-19 L.) } \\
& 138 \mathrm{n} 4 \\
& 61\left(6.270,15^{-17} \text { L. }\right) \\
& \text { 179n44 } \\
& 183 \text { n70 } \\
& 61(6.270,21 \text { L. }) \quad 23 n 4
\end{aligned}
$$

\section{De affectionibus interioribus} 6 (7.182,6 L.) $\quad 189 n 113$ 13 (7.200,20 L.) $\quad 180$ n $47^{2}$ 16 (7.206,4 L.) $\quad 180 \mathrm{n} 47$ $17(7.208,20$ L. $) \quad 180$ n 47 18 (7.212,16 L.) $\quad 18$ on 47 $20 \quad 144 \mathrm{n} 21$ $20(7.216,20-23$ L. $)$ $192 \mathrm{n} 138$ 24 (7.228,12 L.) $\quad 180$ n 47 25 (7.232,3 L.) $180 \mathrm{n} 47$ 26 (7.236,3-7 L.) $192 n 139$ 28 (7.240,11 L.) $\quad 178 \mathrm{n} 38$ 37 (7.258,19 L.) $\quad 178 \mathrm{n} 38$ 39 (7.262,16-18 L.)

$\begin{array}{ll} & 187 \mathrm{n} 102 \\ 41(7.268,20 \text { L. }) & 187 \mathrm{n} 103 \\ 52 \text { (7.298,20 L. }) & 192 \mathrm{n} 145\end{array}$

De alimento 15 (9.102,16 L.) $\quad 305 \mathrm{n} 75$ 23 (9.106,6 L.) $\quad 305 \mathrm{n} 79$ 39 (9.112,3 L.) $\quad 290 n 20,305 n 76$

De arte

$$
\begin{array}{cl}
1\left(6.2,3^{-10} \mathrm{~L} .\right) & 44 \mathrm{n} 13,45 \mathrm{n} 18 \\
& 5^{2 \mathrm{n}} 45 \\
1\left(6.2,15^{-18 ~ L . ~}\right) & 49 \mathrm{n} 34 \\
1.5(6.8,11-19 \text { L. }) & 23 \mathrm{n} 4 \\
2 & 43 \mathrm{n} 13 \\
7(6.10,19-21 \text { L. }) & 48 \mathrm{n} 29 \\
13(6.26,6-9 \text { L. }) & 47 \mathrm{n} 23 \text { and } 24 \\
13(6.26,6-12 \text { L. }) & 49 n 35 \\
\text { De articulis } & \\
63(4.270,14 \text { f. L. }) & 191 \mathrm{n} 128
\end{array}
$$


De capitis vulneribus

13 (3.228,19f. L.) 1910129

De dentitione

$\begin{array}{ll}20 & 92 \\ 30 \text { (8.548,14 ff. L.) } & 93 n 27 \\ 31 \text { (8.548,16 L.) } & 93 n 27 \\ 32 \text { (8.548,18 L.) } & 93 n 27\end{array}$

De diaeta in morbis acutis

(Regimen in acute diseases)

1,2

2 (2.238,3-7 L.) 187ng9

$3 \quad 66$

3 (2.238,8ff. L.) $34 \mathrm{n} 26$

3 (2.240,10-244,1 L.)

$101 n 11$

$4 \quad 144$ n21

8 (2.278,8-280,1 L.)

$15^{2 n} 44$

9-10 (2.298-328 L.)

149n32

$10(2.300,9-302,5$ L.)

187n101

10 (2.302,1 L.) 177 n26

11 (2.302,6 L.) 33n24

12 (2.324,3 f. L.) $33 \mathrm{n} 23$

14 (2.332,3 ff. L.) 179n44

$14\left(2.332,5^{-7}\right.$ L.) 18on $_{51}$

14 (2.332,8 ff. L.) 18on54

14 (2.332,13-334,3 L.) 186ng3

14 (2.334,2 L.) 186n95

14 (2.334,12-14 L.)

179n45

14 (2.334,14-336,1 L.) $182 n 65$

$14(2.334,14-336,5$ L. $)$ 187 ng 8

16 (2.358,1 f. L.) $231 \mathrm{n} 5$

16 (2.358,1f. L.) $\quad 336 \mathrm{n} 6$

17 (2.36o,10 f. L.) $\quad 182 n 65$

17 (2.362,2 L.) $176 \mathrm{n} 15,178$ n29

18 (2.364-376 L.) 140n11

$26 \quad 31,32$ n21, 34n25

$27 \quad 32$

$28 \quad 31$
$32 \quad 24 n 6$

$36 \quad 26,3^{1}$

De diaeta in morbis acutis, Appendix (Appendix to Regimen in acute diseases)

$$
\begin{array}{ll}
14(2.470,8 \text { L. }) & 180 \text { n } 47 \\
17(2.476,11-478,1 \text { L. }) & 178 \text { n } 37 \\
24(2.508,7-9 \text { L. }) & 184 \text { n } 77
\end{array}
$$

De fistulis

3 (6.450,2 L.) $\quad 91 \mathrm{n} 23$

4 (6.450,26 L.) 91023

5 (6.452,16 L.) $91 \mathrm{n} 23$

7 (6.454,16 L.) $192 \mathrm{n} 144$

De flatibus (Breaths)

1 (6.90 L.) 77

1 (6.90,4-6 L.) $\quad 43 \mathrm{n} 13,46 \mathrm{n} 19,55$

$1(6.92,4-5$ L. $) \quad 32$ n22

$1(6.92,11-13$ L. $) \quad 48$ n28

3 (6.94,3 L.) $\quad 49$ n33

3 (6.94,4 L.) $\quad 5$ on 36

3 (6.94,16 L.) $\quad 51$ 1n39

3 (6.94,2-9 L.) $\quad 53$ n46

4 (6.96,1 f. L.) $\quad 5$ 1n 39

5

$125,125 \mathrm{n} 7$ and 8 , $129 n 18$

$6 \quad 58,124,125,124 n 6$

$6(6.96,20-98,13$ L. $)$

6 (6.98,2f. L. $\quad 58$

$7 \quad 127 \mathrm{n} 12$

8 (6.102,22 f. L. $) \quad 5$ on 36

$14 \quad 72,100,100 n 9$

$14(6.110,14$ L. $) \quad 99 n 7$

$15 \quad 73$

15 (6.114,13-17 L.) 47n22

15 (6.114,15-16 L.) 47n24

15 (6.114,13-20 L.) 49n35

De fracturis

$7(3.440,16$ L. $) \quad 182 n 68$

25 (3.498,7 f. .L.) $42 \mathrm{n} 11$

$29(3.516,4$ f. L. $) \quad 1910127$

De glandulis

5 (8.56o,9 L.) $\quad 93$ n28

$12(8.566,3 \mathrm{~L}) \quad 91 \mathrm{n} 23$

$14(8.570,1$ L. $) \quad 91 n 23$ 
De habitu decenti

$$
\begin{gathered}
5(9.232,11-234,1 \text { L. }) \\
281 n_{5} 8 \\
16 \text { (9.242,5-8 L.) } \\
275 \mathrm{n} 39 \\
\text { De haemorrhoidibus }
\end{gathered}
$$

$$
4 \text { (6.440,13 L.) 191n131 }
$$

De humidorum usu (On the Use of

Liquids)

$$
\begin{array}{ll}
3 \text { (6.126,14 L. }) & 93 \mathrm{n} 27 \\
5(6.128,8 \text { ff. L. }) & 190 \mathrm{n} 126 \\
6 & 69 \\
7(6.136,4 \text { f. L. }) & 23 \mathrm{n} 4 \\
6 \text { (6.134,14 L.) } & 91 \mathrm{n} 23
\end{array}
$$

De humoribus

20 (5.500,8ff. L.) $90 \mathrm{on} 21$

De locis in homine

$$
\begin{array}{ll}
29(6.322,8 \text { L. }) & 85,85 \text { n9 } \\
45(6.340,3 \text { ff. L. }) & 32 \mathrm{n} 22 \\
45(6.340,5-7 \text { L. }) & 15^{2 n} 44 \\
47 \text { (6.346,7 L. }) & 192 n 137 \\
47 \text { (6.346,11 L.) } & 192 n 137
\end{array}
$$

De medico

$$
1 \text { (9.204,9-10 L.) 282n64 }
$$

De morbis 1

$\begin{array}{ll}3(6.144,12 \text { L. }) & 234 \mathrm{n} 13 \\ 3(6.144,15 \text { f. L. }) & 235 \mathrm{n} 19 \\ 15(6.166,15 \text { L. }) & 179 n 40 \\ 26(6.192,11 \text { ff. L. }) & 179 n 42 \\ 30 & 233 n 10 \\ 30 \text { (6.200,18f. L. }) & 235 \mathrm{n} 17\end{array}$

De morbis 2

5 (ed. Jouanna, p. 136,14-15)

$75^{\mathrm{f}}$.

$12 \mathrm{f} . \quad 236 \mathrm{n} 22$

$22(7 \cdot 36,14$ L. $) \quad 178 \mathrm{n} 31$

$23(7 \cdot 38,14$ and $16 \mathrm{~L}$.

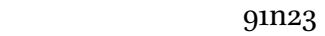

32 (7.48,23 L. $) \quad 1910130$

47 (7.70,17 L.) $191 \mathrm{n}^{2} 2$

$51(7.80,7$ L. $) \quad 182 n 67$

$55(7.86,5$ f. L. $) \quad 179 n 41$

60 (7.94,4 L.) 1910132

72 (7.110,5-10 L.) $140 \mathrm{n} 12$

73 (ch. 62) 236n22

74 (ch. 63) 236n22

\begin{tabular}{|c|c|}
\hline 1 & 122 \\
\hline $1\left(6.35^{2,1} \mathrm{~L}.\right)$ & $\begin{array}{l}47 \mathrm{n} 26 \text { and } 27, \\
99 n 8\end{array}$ \\
\hline 1 (6.352,9 L.) & $128 \mathrm{n} 13$ \\
\hline \multicolumn{2}{|l|}{$1(6.354,3$ and 7 L. $)$} \\
\hline & $128 \mathrm{n} 13$ \\
\hline $1(6.354,4-11$ L. $)$ & 73 \\
\hline $1(6.354,7-10$ L. $)$ & 77 \\
\hline 1 (6.354,12 L.) & $102 n 14$ \\
\hline $1(6.354,12-18$ L. $)$ & 63 \\
\hline $1(6.354,13-14$ L. $)$ & $102 n 16$ \\
\hline $1\left(6.354,15^{-17}\right.$ L. $)$ & $102 n 18$ \\
\hline $1\left(6.35^{8,16-19 ~ L .)}\right.$ & $108 n_{31}$ \\
\hline $1(6.360,10$ L. $)$ & $102 \mathrm{n} 17$ \\
\hline \multicolumn{2}{|c|}{$1(6.360,13-362,6$ L. $)$} \\
\hline & $62,103 \mathrm{f}$ \\
\hline $1(6.362,6-16$ L. $)$ & $108 n_{32}$ \\
\hline \multicolumn{2}{|c|}{$1(6.362,6$ and 13 L. $)$} \\
\hline & $128 \mathrm{n} 13$ \\
\hline \multicolumn{2}{|c|}{$1(6.362,16-364,8$ L. $)$} \\
\hline & 109n34 \\
\hline $1(6.364,8$ L. $)$ & $128 \mathrm{n} 13$ \\
\hline \multicolumn{2}{|l|}{$1(6.362,6-364,8$ L. $)$} \\
\hline & $123 n 4$ \\
\hline $2(6.364,11$ f. L. $)$ & 64 \\
\hline 3 & $43 n 13$ \\
\hline 5 & $236 n 20$ \\
\hline 7 & 10ong \\
\hline 7 (6.372,5 ff. L.) & 72 \\
\hline 9 & 73 \\
\hline $11(6.382,8-11$ L. $)$ & 64 \\
\hline
\end{tabular}

De morbis 3

1 (7.118,17 L. $) \quad 182 n 64$
2 (7.120,7 L.) 182 n64

$3(7.122,3$ L. $) \quad 182 n 64$

4 (7.122,14 L.) $\quad 182$ n64

5 (7.122,21 L. $\quad 182 n 64$

7 (7.126,15 L.) $\quad 182 n 64$

$8(7.128,2$ L. $) \quad 182 n 64$

$10(7.130,14 \mathrm{~L}) \quad .182 \mathrm{n} 64$

17 (7.16o,5 L.) 179n47, 189n111

De morbis 4

$$
\begin{array}{ll}
4 & 44 \mathrm{n} 13 \\
12 & 43 \mathrm{n} 13 \\
32,1(7 \cdot 542,6-9 \text { L. }) & \\
\multicolumn{3}{c}{336,336 \mathrm{ng}}
\end{array}
$$

57 (ch. 26) (7.612 L.)

$43 \mathrm{n} 12$

De morbo sacro 


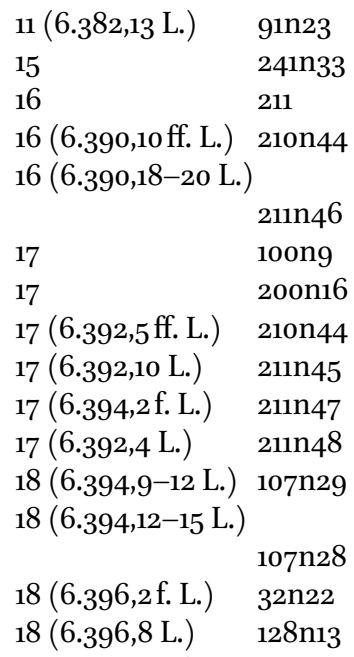

De mulierum affectibus III = De sterilitate (On Sterile Women)

\begin{tabular}{|c|c|}
\hline & 5 \\
\hline $218(8.422,18-20$ & \\
\hline & $184 \mathrm{n} 74$ \\
\hline e natura hominis & \\
\hline 1 (p. 166,11 Jouan & \\
\hline & $327 \mathrm{n} 17$ \\
\hline $1(6.32,1-3$ L. $)$ & $44 \mathrm{n} 14$ \\
\hline $1-8$ & 321 \\
\hline 2 & $44 \mathrm{n} 13$ \\
\hline $2(6.36,2-4$ L. $)$ & $296 n 38$ \\
\hline $2(6.34,6$ L. $)$ & $298 \mathrm{n} 45$ \\
\hline 4 & $43 n 13,230 n 1$ \\
\hline $4(6.38,19-40,6 \mathrm{~L}$ & \\
\hline & $23,23 n 6$ \\
\hline 4,1 & $335^{\mathrm{n} 1}, 343^{\mathrm{n}} 27$ \\
\hline $4,2-3$ & $335 n 3$ \\
\hline 5,1 & $343 n 27$ \\
\hline 7 & $335^{\mathrm{n} 2}$ \\
\hline 7 & 23on2, 149n34 \\
\hline 8 & $231 n_{7}$ \\
\hline 9 & $60,126 \mathrm{n} 10,127 \mathrm{n} 11$ \\
\hline $9\left(6.5^{2,8}-9\right.$ L. $)$ & $3^{2 n 22}$ \\
\hline $9(6.54,1-4$ L. $)$ & 143n20 \\
\hline $9(6.54,14-17$ L. $)$ & $140 n 12$ \\
\hline $9-15$ & 321 \\
\hline 15,5 & $335 n_{5}$ \\
\hline 16 & $335^{n} 4$ \\
\hline 15 & $230 n 3,232 \mathrm{n} 8$ \\
\hline 16 & 188 n106 \\
\hline $16(6.72,1-5$ L. $)$ & 15on35 \\
\hline $16(6.72,5$ ff. L. $)$ & $150 n 37$ \\
\hline $16(6.72,10-74,4)$ & \\
\hline & 15 on 36 \\
\hline $16(6.74,4-8$ L.) & 150 37 \\
\hline $16\left(6.74,9^{-13}\right.$ L. $)$ & 15 on35 \\
\hline 19 & $144 \mathrm{n} 21$ \\
\hline 20 & 187ng6 \\
\hline 21 & $181 n_{57}, 181 n 63$ \\
\hline 22 & $144 n 21$ \\
\hline e natura muliebri & Nature of Women) \\
\hline 5 (7·318,4f. L. $)$ & $191 n 135$ \\
\hline 18 (7·338,17 L.) & $128 \mathrm{n} 13$ \\
\hline $33(7 \cdot 366,8-370$, & L.) \\
\hline & $191 n 136$ \\
\hline 59 (7.398,12 L.) & $184 \mathrm{n} 78$ \\
\hline
\end{tabular}


$\begin{array}{ll}\text { De natura ossium } & \\ 12(9.184,9 \text { L. }) & 93 n 28 \\ 13(9.184,14 \text { L.) } & 93 \text { n28 } \\ 16 & 93 n 28 \\ 17 & 93 n 28 \\ 18 & 93 n 28\end{array}$

De octimestri partu (Eight months child) $2 \quad 43 n 13$

De semine/De natura pueri
1 (7.470,1 L.)
$45^{\mathrm{n} 17}$
4 (7.476,15f. L.)
6
$43 n 12$
(5. 43 (7.496,gf. L.
15 (7.496,9f. L.) $\quad 43 \mathrm{n} 12$
18 (7.504,2 f.L.) $\quad 44 \mathrm{n} 15$
32 (7.542,1-2 L.) $\quad 46 \mathrm{n} 21$

De ulceribus

$\begin{array}{ll}3(6.404,14 \text { L. }) & 91 n 23 \\ 10(6.410,2-3 \text { L. }) & 90,93, \text { 9on21, } \\ & 91 n 23 \\ 11(6.410,23 \text { L. }) & 192 n 141 \\ 18 & 92\end{array}$

De vetere medicina

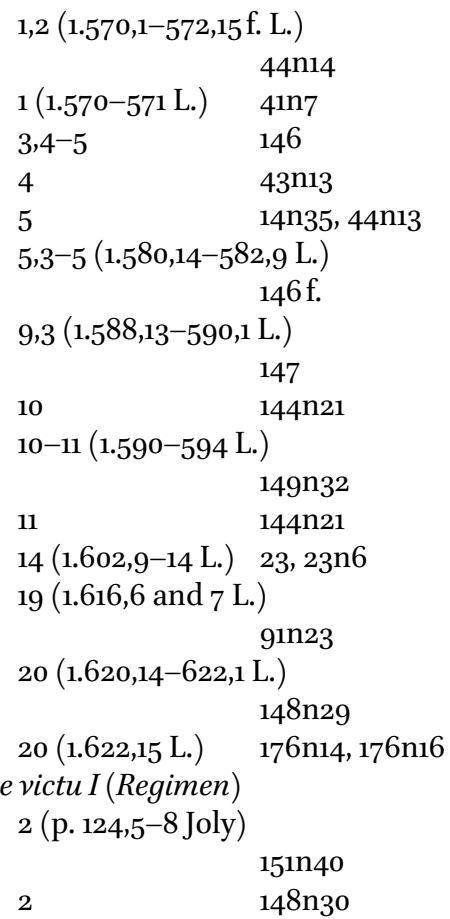

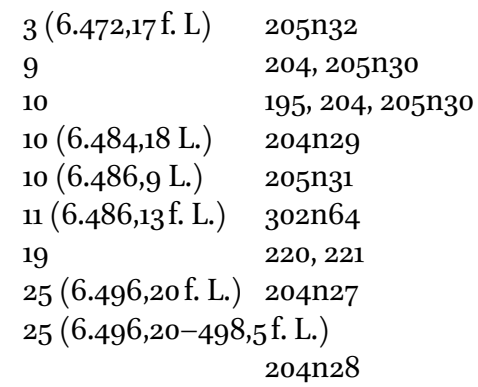




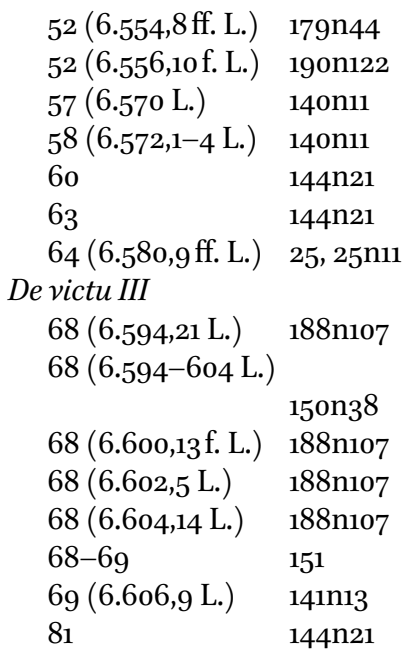

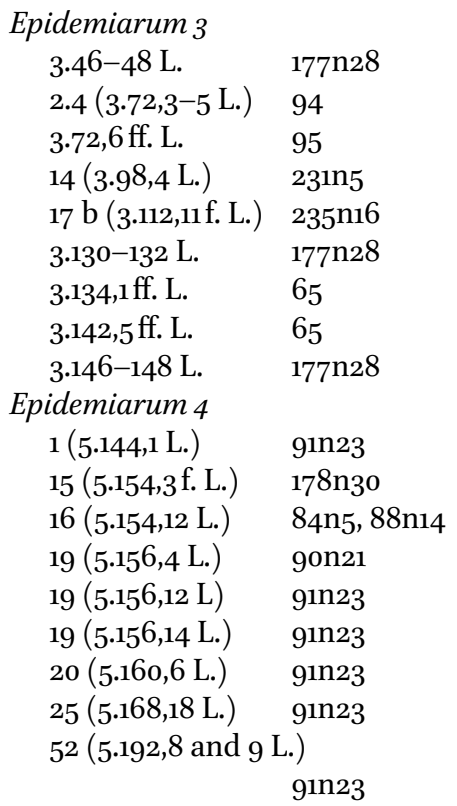

\section{Epidemiarum 5}

$$
\begin{array}{ll}
2 \text { (5.204,7 L. }) & 178 \mathrm{n} 30 \\
44(5.234,1 \text { L. }) & 90 \mathrm{n} 21 \\
71(5.244,20 \text { f. L. }) & 178 \mathrm{n} 36 \\
86(5.252,11 \text { L. }) & 176 \mathrm{n} 17 \\
86(5.252,11-15 \text { L. }) & \\
& 178 \mathrm{n} 32
\end{array}
$$

\section{Epidemiarum 6}

$$
\begin{aligned}
& 1.11 \text { (5.272,1 L.) } 84 \mathrm{n}_{5}, 86 \mathrm{nn} 1 \text {, } \\
& 88 \mathrm{n} 14 \\
& 2.6 \text { (5.280,5 L. }) \quad 84 \mathrm{n}_{5}, 85,86 \\
& 2.11(5.282,16 \text { L. }) \quad 84 \mathrm{n} 5 \\
& 2.11(5.282,16 \mathrm{~L} .) \quad 85,86 \\
& 2.24 \text { (5.290,4-6 L.) } \\
& 272 n 35 \\
& 3.23 \text { (5.304,3 L.) 9on } 21 \\
& 4.7(5 \cdot 308,15 \text { f. L. }) \quad 268 \mathrm{n} 20 \\
& 4 \cdot 7 \text { (5.308,14-15 L.) } \\
& 270 \mathrm{n} 25 \\
& \text { 5,1 (5.314,5 L.) } 290 n 19 \\
& \text { 5,1 (5.314,7-8 L.) } \quad 305^{\mathrm{n}} 77 \\
& 5 \cdot 7(5 \cdot 318,1-4 \text { L. }) \quad 272 n 33 \\
& 7(5 \cdot 326,14-328,1 \text { L. }) \\
& 184 \mathrm{n} 76 \\
& 7 \text { (5.328,2 f. L. }) \quad 181 n 60 \\
& 8.31(5 \cdot 354,19-356,3 \text { L.) }
\end{aligned}
$$




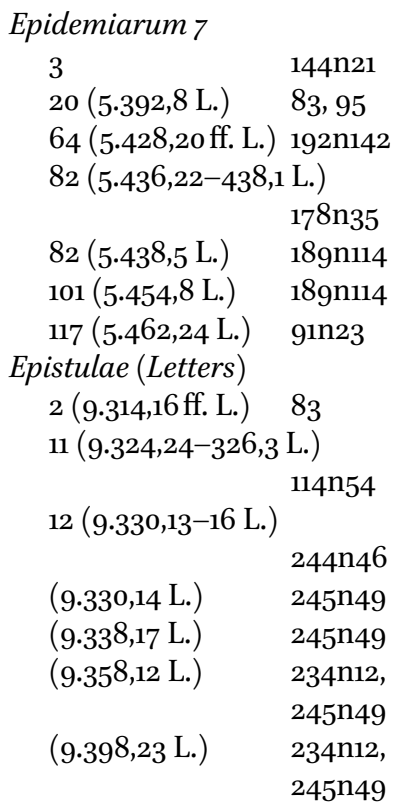

Hippocratis ius iurandum (4.628,1 L.) 68n19, $113 n_{51}$

Praeceptiones

$$
\begin{array}{ll}
6 \text { (9.258,5-8 L. }) & 278 \mathrm{n} 50 \\
6 \text { (9.258,10 L. }) & 282 \mathrm{n} 63
\end{array}
$$

Prognosticon

$$
\begin{array}{ll}
(2.110,1 \text { L. }) & 302 n 63 \\
1(2.112,3-6 \text { L. }) & 109 n 36 \\
10(2.134,5 \text { L. }) & 296 n 37 \\
11(2.136,7-9 \text { L. }) & 88 \mathrm{n} 14 \\
15(2.150,14 \text { f. L. }) & 273 n 36 \\
25(2.190,2 \text { f. L. }) & 273 n 36
\end{array}
$$

Prorrheticon 1

$$
\begin{array}{ll}
26(5.516,9 \text { L. }) & 84,84 \mathrm{n} 5 \\
123\left(5 \cdot 55^{2,5} \text { f. L. }\right) & 84 \mathrm{n}_{5}, 235 \mathrm{n} 18
\end{array}
$$

Prorrheticon 2

$$
\begin{array}{ll}
1 \text { (9.6,14 L. }) & 273 \mathrm{n} 37 \\
1 \text { (9.6,19 L.) } & 27 \mathrm{n} 14 \\
4(9 \cdot 20,10 \text { f. L. }) & 44 \mathrm{n} 13 \\
12 & 92 \\
13 & 92,94 \mathrm{n} 30 \\
13 \text { (9.36,6 ff. L.) } & 93
\end{array}
$$

Thessali legati oratio = Epistula 27 (9.414,3-9 L.) $\quad 67 \mathrm{n} 12,114 \mathrm{n}_{5} 6$ $(9.416,17$ L. $) \quad 116 \mathrm{n} 62$

$\begin{array}{ll}\text { Homer } \\ \text { Iliad } \\ \text { I,50-52 } & \\ \text { I,61 } & 58 \\ \text { II,731 } & 56,60 \\ \text { XI,514-515 } & 9,17 \mathrm{n} 43 \\ \text { IV,219 } & 9 \\ \text { XXIII,177 } & 93 \\ \text { XXIII,181 } & 93 \\ \text { Odyssey } & \\ 4,125 \mathrm{f} . & 8 \\ 4,219 \mathrm{ff} . & 173 \\ 4,227-232 & 8 \\ 9,208 & 189 n 112 \\ 10,234-236 & 189 n 115 \\ 10,302-304 & 325,3^{28} \\ 21,294 & 183 n 71\end{array}$

Iamblichus

De vita Pythagorica

175

$35 \mathrm{n} 28$

Isidore of Seville

De numeris

23

$249 n 62$

Isocrates

Busiris

$21-22$

11n23, 15n37

Leucippus

DK 67 A $30 \quad 212 n_{50}$

Lucretius

De rerum natura

$\begin{array}{ll}6,1095^{-1101} & 133 n 22 \\ 6,1129 f . & 133 n 22\end{array}$

Macrobius

Saturnalia

$7.12,25 \mathrm{f}$.

$170 n 19$

Nemesius of Emesa

De natura hominis
7
$292 n 25$
p. 44,24 Morani $356 \mathrm{n}_{52}$
p. $45,4-6$ Morani $356 n_{52}$ 
Oribasius

Collectionum Medicarum Reliquiae 5.3 (pp. 117-120 Raeder)

5.3.9 (p. 118,2 Raeder) $17 \ln 21$

$129 n 17$

$5 \cdot 3$ (p. 118,11-16 Raeder) 166n11

$5 \cdot 6,5^{-7} \quad 188 \mathrm{n} 104$

$5 \cdot 7 \quad 177 \mathrm{n} 23$

5.7.1-2 173n1

lib. inc. 1,4-6 340n21

Palladius

Commentary on Hippocrates'

Epidemics 6

Dietz II 2,18-23 125n9, 136n27

Pausanias

Descriptio Graeciae

10.2.6

${ }^{115} \mathrm{n}_{5} 8$

Philoponus

De aeternitate mundi

p. 283,19 Rabe $\quad 306 \mathrm{n} 80$

In Aristotelis de generatione et corruptione

p. 106,33 Vitelli 306 n8o

Pindar

Fourth Pythian Ode

$270 \quad 21,21 n 1$

Plato

Alcibiades II

140c-e 200015

Gorgias

$45^{6 \mathrm{~b}}$

Leges

666a-c

$747 \mathrm{~d}$

797 dff.

798

Lysis

219e

Meno

$76 \mathrm{a}$

$$
21,211
$$

oon15

39n2, 51, 79n41

$18 \ln _{5} 8$

170

36

$36 \mathrm{n} 30$

$190 n 120$

$213 n 5^{2}$

\begin{tabular}{|c|c|}
\hline \multirow{2}{*}{\multicolumn{2}{|c|}{$\begin{array}{l}76 \mathrm{c}-\mathrm{d} \\
\text { Phaedo }\end{array}$}} \\
\hline & \\
\hline $96 a-b$ & 200 \\
\hline $96 \mathrm{a}_{5} \mathrm{ff}$. & $298 n 48$ \\
\hline \multicolumn{2}{|l|}{ Phaedrus } \\
\hline $266 e-267 c$ & $40 n_{4}$ \\
\hline $267 \mathrm{~d}$ & 46 \\
\hline $270 \mathrm{~b}$ ff. & $39 n 3$ \\
\hline $270 \mathrm{c}$ & $299 n_{50}$ \\
\hline $270 \mathrm{c}-\mathrm{d}$ & 325 \\
\hline \multicolumn{2}{|l|}{ Politicus (Statesman) } \\
\hline $288 \mathrm{e} 5$ and $289 \mathrm{~b} 1$ & 327 \\
\hline $287 \mathrm{~d}$ & 327 \\
\hline \multicolumn{2}{|l|}{ Protagoras } \\
\hline $311 b-c$ & $\begin{array}{l}55^{\mathrm{n} 1}, 68 \mathrm{n} 18 \\
278 \mathrm{n} 49\end{array}$ \\
\hline $322 \mathrm{~b}$ & $96 \mathrm{n} 32$ \\
\hline \multicolumn{2}{|l|}{ Res publica } \\
\hline $404 a$ & $27 \mathrm{n} 14$ \\
\hline $405 \mathrm{~d}-406 \mathrm{a}$ & $14 n_{3} 6$ \\
\hline \multicolumn{2}{|l|}{ Symposium } \\
\hline $176 c-d$ & $177 \mathrm{n} 20$ \\
\hline 186b1-3 & 51 \\
\hline $188 \mathrm{~b}$ & 218 \\
\hline 186diff. & $32 \mathrm{n} 22$ \\
\hline \multicolumn{2}{|l|}{ Theaetetus } \\
\hline $194 c$ & $207 n 38$ \\
\hline \multicolumn{2}{|l|}{ Timaeus } \\
\hline $34 a$ & 220,221 \\
\hline $36 c$ & 220,221 \\
\hline $42 \mathrm{C}$ & 220,221 \\
\hline $43^{b}-44 b$ & 222 \\
\hline $43^{b-c}$ & 224 \\
\hline $43 c$ & 221 \\
\hline $43 d$ & $220,221,223,224$ \\
\hline $44 \mathrm{a}$ & 221,225 \\
\hline $44 b-c$ & 221,223 \\
\hline $47 d$ & 220,221 \\
\hline $67 c-d$ & $213 n_{52}$ \\
\hline $86 e-87 a$ & 220,223 \\
\hline \multicolumn{2}{|l|}{$\begin{array}{l}\text { Pliny the Elder } \\
\text { Historia naturalis }\end{array}$} \\
\hline 7.114 & $19 n 49$ \\
\hline 7.123 & $175^{\mathrm{n} 8}$ \\
\hline $14.5^{8}$ & 190n120, 190n125 \\
\hline 14.73 & 18on49 \\
\hline
\end{tabular}




\begin{tabular}{|c|c|}
\hline \multicolumn{2}{|c|}{ Historia naturalis (cont.) } \\
\hline 14.80 & 19on121, 190n124 \\
\hline $23 \cdot 31$ & $175^{\mathrm{n} 11}$ \\
\hline $23 \cdot 31-51$ & $174 \mathrm{n} 6$ \\
\hline $23 \cdot 32$ & 175ng, 187n10o \\
\hline $23 \cdot 37$ & $184 \mathrm{n} 82$ \\
\hline $23 \cdot 38$ & $175^{\mathrm{n} 10}$ \\
\hline 23.43 & $190 n 120$ \\
\hline $23.5^{0}$ & $184 \mathrm{n} 83$ \\
\hline $23 \cdot 5^{1}$ & $189 n 110$ \\
\hline 37.123 & $83 n 3$ \\
\hline
\end{tabular}

Plutarch

De tuenda sanitate praecepta 19,132 b $\quad 183 n 69$

Vita Lysandri

$\begin{array}{ll}1.2,5(175 \mathrm{~b}-\mathrm{c}) & 239 \mathrm{n} 27 \\ 1.28,1 & 239 \mathrm{n} 28\end{array}$

Vita Periclei

$\begin{array}{ll}1.6,154 \mathrm{f} .-155^{\mathrm{a}} & 64 \\ 171 \mathrm{a} & 94 \mathrm{n} 30\end{array}$

Quaestiones convivales

$\begin{array}{ll}3.1,647 \mathrm{a} & 183^{n} 69 \\ 735^{\mathrm{b}} & 212\end{array}$

Polybius

Historiae

$1.81,5^{-10}$

$96 n 33$

Pollux

Onomasticon

4

9102

Proclus

Commentary on Timaeus

Diehl 3.332,3-16 226

Rufus of Ephesus

De melancholia

frag. 70

De vino

frag. I § $2 \quad 177 \mathrm{n} 24$

On the Names of the Parts of the

Human Body

133-134

2on5o
Sextus Empiricus

Outlines of Pyrrhonism

$1.5^{1}$

357n55

Sophocles

Aiax

447

Antigone

$172 \quad 121 n 2$

$776 \quad 121 n^{2}$

$1015 \quad 123 n 5$

$1042 \quad 121 n 2$

1043f. $\quad 123 n_{5}$

$1113 \mathrm{f} . \quad 35^{\mathrm{n} 28}$

Oedipus Rex

22-30 57

27-30 124

97

$99 \quad 124$

100-107 $\quad 58$

$101 \quad 124$

141-147 62

164-166 61

$168 \mathrm{ff} . \quad 57$

$241 \quad 58,121 n 2$

$313 \quad 58,121 n 2$

$387 \quad 102$

$388 \quad 102$

$405 \quad 103$

$1012 \quad 58,121 n 2$

Philoctetes

7

$184 \mathrm{ff} . \quad 82$

$226 \quad 82$

$265 \quad 82$

$313 \quad 90 n 19$

677-699 $\quad 88$

$695 \quad 90 n 19$

$697 \quad 89$

$745 \quad 90 n 19$

$919 \quad 68$

1326-1334 68

1437-1438 67

Trachiniae

$\begin{array}{ll}573 \text { f. } & 232 \text { n1o } \\ 77 \text { of. } & 71 \\ 794 & 73\end{array}$




\begin{tabular}{|c|c|c|c|}
\hline 987 & $90 n 19$ & \multicolumn{2}{|c|}{ Historia plantarum } \\
\hline 1026 & 82 & $9.11,3$ & $99 n_{5}$ \\
\hline 1030 & 82 & 9.15 & 9 \\
\hline 1054 & $89,90 n 19$ & & \\
\hline 1084 & $89,90 n 19$ & Thucydides & \\
\hline 1088 & $90 n 19$ & 1.20 & 25 \\
\hline & & 1.42 & $23 n 4$ \\
\hline Soranus & & $1.71 \cdot 3$ & $35 \mathrm{n} 28$ \\
\hline De mulierum & & $2.47 \cdot 4$ & $135^{\mathrm{n} 24}$ \\
\hline 1.38 & $179 n 43$ & 2.48 .1 & 132 \\
\hline 1.46 & $185 \mathrm{n} 88$ & 2.48 .3 & $3^{1 n 21,} 3^{2 n 21,} 131$ \\
\hline 1.54 & $185 \mathrm{n} 89$ & 2.51 .2 & $23 n 4$ \\
\hline 1.64 & 186ngo & 2.51 .4 & $135^{\mathrm{n} 24}$ \\
\hline 2.26 & 186ng1 & $2.5^{2.4}$ & $59 n 6$ \\
\hline & & 2.54 .5 & $94 n 30$ \\
\hline Stephanus & & $3 \cdot 3 \cdot 3$ & $35^{\mathrm{n} 28}$ \\
\hline $\begin{array}{l}\text { Commentar } \\
\text { therapeuticu }\end{array}$ & $\begin{array}{l}\text { em Galeni librum } \\
\text { auconem }\end{array}$ & 6.13 & $\begin{array}{l}28 \mathrm{n} 16,29,29 n 17 \text {, } \\
34 \mathrm{n} 25\end{array}$ \\
\hline $1.220,5$ & $306 n 80$ & 6.14 & $21,22,28,28 \mathrm{n} 15$ \\
\hline 1.262 & $185 \mathrm{n} 85$ & & $28 \mathrm{n} 16,15^{2 n} 44$ \\
\hline In Hipp. Proc & & 6.18 .6 & $23,25,28,28 \mathrm{n} 16$ \\
\hline 1.1 & $35^{8 n} 59$ & & $29 n 17,31,26$ \\
\hline & & 6.18 .7 & $26,27 \mathrm{n}_{15}, 28 \mathrm{n} 16$ \\
\hline Stobaeus & & & $3^{0,15^{2 n} 44}$ \\
\hline $1.49,53$ & $218 n 62$ & 7.87 & $32 \mathrm{n} 21$ \\
\hline & & 7.87 .1 & $32 \mathrm{n} 21$ \\
\hline Strabo & & & \\
\hline Geographia & & Xenophanes & \\
\hline $14.2,19$ & $113 n_{50}$ & DK 21 A 46 & $163 n 9$ \\
\hline Theognis & & Xenophon & \\
\hline $211-212$ & $183 n 71$ & Cyropaedia & \\
\hline & & $8.1 .3^{8}$ & $144 \mathrm{n} 22$ \\
\hline Theophrast & & 8.8 .9 & 144 \\
\hline Characters & & De republica $L c$ & emoniorum \\
\hline $19 \cdot 3$ & $85^{n} 7$ & 2,5 & $143 n 19$ \\
\hline De sensibus & & 5,3 & $143 n 19$ \\
\hline 10 & $219 n 66$ & Hellenica & \\
\hline ch. $10-11$ & $214 \mathrm{f}$. & $6.4,18$ & $24 n 6$ \\
\hline 20 & 212 & & \\
\hline
\end{tabular}

